Cahiers d'études italiennes

\title{
Identità femminile e generazionale in In principio erano le mutande di Rossana Campo
}

Peter Gahl

\section{OpenEdition}

\section{Journals}

Edizione digitale

URL: http://journals.openedition.org/cei/917

DOI: $10.4000 /$ cei. 917

ISSN: 2260-779X

\section{Editore}

UGA Éditions/Université Grenoble Alpes

\section{Edizione cartacea}

Data di pubblicazione: 15 maggio 2008

Paginazione: 137-148

ISBN: 978-2-84310-121-2

ISSN: $1770-9571$

Notizia bibliografica digitale

Peter Gahl, «Identità femminile e generazionale in In principio erano le mutande di Rossana Campo », Cahiers d'études italiennes [Online], 7 | 2008, Messo online il 15 novembre 2009, consultato il 01 mai 2019. URL : http://journals.openedition.org/cei/917 ; DOI : 10.4000/cei.917 


\title{
IDENTITÀ FEMMINILE E GENERAZIONALE IN IN PRINCIPIO ERANO LE MUTANDE DI ROSSANA CAMPO
}

\author{
Peter Gahl \\ Université de Constance
}

Le profonde trasformazioni che si sono prodotte nella società italiana a partire dagli anni ' 80 e i noti rovesciamenti politici verificatisi a cavallo tra la fine dello stesso decennio e l'inizio del successivo hanno non solo messo in crisi alcune certezze ideologiche dei decenni precedenti, ma hanno anche - e non poteva essere altrimenti - inciso sul rapporto tra i due sessi. Il presente contributo è dedicato ad una scrittrice che si è affermata soltanto dopo i mutamenti suddetti : si tratta di Rossana Campo, nativa di Genova, classe '63, ma che da qualche anno a questa parte vive a Parigi. L'autrice fa parte di quel filone eterogeneo della letteratura italiana recente che Renato Barilli qualifica come "la terza ondata", ovvero come la "neoneoavanguardia» : per essere più precisi, essa appartiene non alla famosa ma effimera "Gioventù cannibale», bensì a quel gruppo più addomesticato dei "giovani narratori", tutelati da ex membri del Gruppo 63 come Nanni Balestrini e Gianni Celati. Lo scopo di queste pagine è di chiarire come si articola la coscienza dell'identità femminile e generazionale nel suo romanzo d'esordio, intitolato In principio erano le mutande e pubblicato dalla Feltrinelli nel 1992. Ho scelto questo romanzo perché risulta a mio avviso più significativo di altre opere in relazione alla questione che ci interessa. A parte ciò, questo testo è a detta della stessa autrice - anche a distanza di parecchi anni - tra quelli che le stanno più a cuore ${ }^{1^{*}}$.

In principio erano le mutande presenta caratteristiche riconducibili al genere picaresco : la narratrice e protagonista è una donna ventisettenne senza mestiere ben preciso che fa parte di una sorta di bohème genovese. La giovane tira avanti a forza di prestiti, lavoretti e inviti, e gran parte del testo racconta la sua movimentata vita sentimentale (e sessuale) : la giovane si considera afflitta da "una lunga serie infinita di sfighe ${ }^{2}$ », e - pur essendosi dedicata «alla ricerca dei piaceri edonistici sfrenati di vera lussuria» (PM 28) - si innamora ripetutamente di tipi definiti ogni volta

* Notes p. 147.

Cahiers d'études italiennes, $\mathrm{n}^{\circ}$ 7, 2008, p. 137-148. 137 
come «infami». Il romanzo è tuttavia a lieto fine, perché a un certo punto la giovane si ritrova incinta e riesce a convincere il padre del nascituro «l'infame numero tre» (PM 55) - a mettere su casa con lei.

All'interno della trama sono inoltre inseriti dei capitoli basati su ricordi della prima adolescenza della narratrice. Si tratta tuttavia di episodi non legati alla vicenda principale : nel complesso la struttura del libro risulta dunque variegata e in certo qual modo discontinua, complicata anche dall'inserzione di digressioni relative a personaggi secondari o al racconto di antefatti, ricordi ecc. Tali digressioni sono di regola introdotte da formule colloquiali come : "Ora la storia di questa Christina ve la faccio" (PM 63). Pertanto l'andamento della narrazione piuttosto che seguire il lineare svolgimento diacronico dei fatti è costruito sulla falsariga del racconto casuale, in cui l'intreccio dei vari fili viene gestito in modo da apparire frutto di un'affabulazione spontanea e naturale.

Per poter mettere in evidenza quanto questo romanzo sia legato a un momento storico ben preciso, potrà essere utile ricorrere talvolta al confronto con un testo per certi versi affine ma che risale a un'epoca diversa, e cioè il famoso Porci con le ali (1976), apparso alla vigilia delle contestazioni del Settantasette e scritto a quattro mani da Lidia Ravera e Marco Lombardo Radice (che si celano però dietro lo pseudonimo di «Rocco e Antonia»). Questo libro, oltre a essere uno dei successi editoriali più strepitosi del periodo (partendo da una tiratura iniziale di seimila copie si arrivò a nove ristampe con un totale di 300 mila esemplari già nei primi sei mesi, e ormai il numero di copie vendute ha superato la soglia dei due milioni), può essere considerato emblematico della cultura giovanile di quell'epoca almeno quanto i jeans a zampa d'elefante o certi dischi dei Pink Floyd e di Lucio Battisti. Porci con le ali racconta in forma dialogica le vicende di due liceali a Roma che fanno parte di un gruppo della sinistra extraparlamentare studentesca. Quei due ragazzi, tra riunioni assemblee manifestazioni varie, s'innamorano, si amano, si separano, fanno altre esperienze del genere. Il libro intende proporsi come un contributo al "dibattito sulla sessualità», e infatti questo è l'argomento principale. Dunque, notevole affinità tematica con l'esordio letterario della Campo.

\section{«Oralità scritta» ?}

Parecchi dei critici - militanti e accademici - che si sono occupati di In principio erano le mutande e dei libri successivi di Rossana Campo sostengono che la loro originalità (ammessa o contestata che sia) risieda in prima 
linea nella forma linguistica, che sarebbe riconducibile all'intenzione di riprodurre a livello lessicale e sintattico un certo modo di parlare giovanile e gergale. Questo è il punto di partenza della violenta stroncatura di Roberto Cotroneo ne L'Espresso (14 giugno 1992), il quale cita a titolo d'accusa ben quattro frasi a suo parere particolarmente orrende ${ }^{3}$. Ma sulla pretesa autenticità linguistica dell'autrice insistono anche contributi esenti da una vena polemica, come quello di Marco Berisso ${ }^{4}$ che include il romanzo in un campionario di quindici testi narrativi scritti da giovani autori nei primi anni '90, sottoponendolo a un'analisi stilistica comparata, oppure quello di Giulio Conti che discute le soluzioni addottate nelle finora cinque traduzioni tedesche della narrativa campiana, definendola nello stesso titolo del suo saggio come "oralità scritta ${ }^{5}$ ".

Che le cose non stiano semplicemente così è l'autrice stessa a dirlo. In un'intervista rilasciata a Kate Litherland nel 1999 dichiara, è vero, il suo ripudio di una «lingua accademica, artificiale e paludata ${ }^{6}$ » e afferma che il suo ideale sia " una letteratura che faccia i conti con la forza e l'energia del parlato $^{7}$ ». Ma in seguito ci tiene a precisare : «Io non è che utilizzavo il gergo giovanile nel senso che vado in giro col microfono e poi sbobino. La mia lingua è sempre un'elaborazione letteraria dell'idea della lingua parlata $^{8} »$. E seguendo questa sua intenzione che l'autrice varia sensibilmente il proprio stile di libro in libro. La cifra stilistica dominante in In principio erano le mutande riproduce certamente un'oralità "bassa» e giovanile, ma ogni tanto si avverte pure un certo distacco ironico da quella matrice linguistica. Basti un esempio, preso dalla prima pagina del libro : la protagonista si sente assediata dal suo amico Luca che insiste "per riavere le sue trecentomila lire. I miei trecento sacchi, dice lui così ».

Inoltre, più che da uno solo, il romanzo è caratterizzato (più di ogni opera successiva dell'autrice) dalla copresenza e dall'intrecciarsi di registri diversi e tra di loro contrastanti. Per esempio, l'autrice fa uso ogni tanto di elementi tipici del linguaggio burocratico come la posposizione del numerale («fidanzato da anni sette», PM 42) e la sostituzione della prima persona con la locuzione "la sottoscritta». Un effetto simile risulta da strutture sintattiche ridotte che ricordano vagamente lo stile telegrafico, attraverso la soppressione del verbo e dell'articolo. Certo, queste due peculiarità stilistiche sono abbastanza diffuse anche nelle conversazioni e narrazioni familiari ; fanno parte di certo umorismo colloquiale e neppure esclusivamente giovanile, e perciò possono, volendo, considerarsi motivate dall'intento di servirsi di un linguaggio "orale». Ciò non vale invece per altre caratteristiche della prosa del libro : parecchie volte sono imitati espedienti narrativi tipici della letteratura per l'infanzia, come per esem- 
pio i titoli dei capitoli che ne riassumono il contenuto9 ${ }^{9}$ Ma non sono infrequenti nel testo anche interventi metanarrativi di altri tipi, che non rimandano necessariamente alla tradizione del libro per l'infanzia.

Più importante ancora mi pare la presenza di un'altra categoria di elementi che non derivano dal linguaggio gergale : mi riferisco a intere sequenze in cui tale linguaggio, pur presente, è contaminato con palesi rimandi a situazioni topiche tratte dalla letteratura romanzesca dell'Ottocento. Ad esempio l'intera sequenza del primo incontro d'amore, che avviene tra gli scaffali di celebri opere ottocentesche, come Cime tempestose e la Certosa di Parma : nella soffitta dell'artista-pittore i due innamorati finiscono per sentirsi «esatti esatti Rodolfo e Mimì» (PM $57)^{10}$.

Per riassumere : lo scopo principale delle scelte stilistiche che caratterizzano i vari romanzi campiani non è quello di riflettere mimeticamente una determinata realtà linguistica ${ }^{11}$, ma quello di creare, attraverso il ricorso sistematico al racconto in prima persona, un'immagine il più possibile coerente di protagonisti principali spesso caratterizzati da una personalità vistosamente incoerente, com'è il caso dell'eroina di In principio erano le mutande.

\section{Un romanzo postideologico}

L'incipit di In principio erano le mutande è caratterizzato dalla volontà della narratrice di mettere subito in chiaro alcune cose : il primo capoverso allude ben due volte alle sue ristrettezze economiche (menziona «le mie borse della spesa non pesanti», PM 11, e un debito che lei non è in grado di saldare) ; inoltre si fa menzione anche di una certa Akofa e l'eroina si dichiara "abituata alle donne africane mie vicine di casa» (ibid.). Le preme ovviamente di segnalare sin dall'inizio che la storia è ambientata nella società italiana multietnica e trasformata - quella degli anni novanta appunto - e che la protagonista abbia familiarità con quella sottocultura particolare. È significativo che né quella Akofa, né alcun'altra donna africana compaiano nel seguito del libro : ciò dimostra che questa battuta assolve unicamente la funzione di un temporal marker.

Ma il testo offre anche altri esempi di elementi simili, che servono a segnalare che l'Italia non è più quella che era stata fino a pochi anni prima. A volte sono i personaggi stessi ad alludere a un loro passato nettamente distinto dalla condizione presente. Nel settimo capitolo, la «amica del cuore Giovanna» esprime un giudizio scettico su un nuovo amante della 
protagonista e dichiara : "Lo conosco da un po' io sai, aveva una storia con una che faceva politica con me» (PM 43). Il «fare politica insieme» è diventato dunque un fatto che appartiene a un'epoca irrevocabilmente passata. Certe ideologie che caratterizzavano quell'epoca sono rievocate di passaggio anche da una specie di running gag, una battuta che ricorre più volte nel romanzo : di tanto in tanto la protagonista si procura degli indumenti di cui ha bisogno tramite un cosiddetto "esproprio rivoluzionario " (vale a dire : un furto) ai danni dei grandi magazzini (PM 55, 63). Una volta rincara persino la dose : «Così pensiamo di fare spedizione da Coin per ennesimo esproprio rivoluzionario antiapartheid pure» (PM 118). Si potrebbe supporre che l'autrice miri a tacciare di indifferenza morale la generazione presente, pronta persino a prendere in giro gli ideali di una volta. Sembra più plausibile invece un'interpretazione opposta : questa battuta sul conto dei rivoluzionari di vent'anni prima denuncia l'ipocrisia e la presunta superiorità morale di chi all'epoca pretendeva di potersi procurare tutti gli oggetti di consumo desiderati senza spendere una lira, in più con la coscienza di chi rivendica i diritti delle classi sfruttate. È interpretabile nello stesso senso anche la spiritosa descrizione di Marco, un amico della protagonista, che ha conosciuto la sua fidanzata «quest'inverno durante l'occupazione dell'università che tutti occupavano felici e ci davano dentro a organizzare i famosi seminari autogestiti [...]. Marco lui è già laureato da un pezzo ma appena c'era aria di occupazione autogestione seminari lui si è lanciato e si dava anzi un sacco da fare» (PM 128).

Questi commenti maligni, oltre a confermare che si tratta di un libro datato, inequivocabilmente legato all'epoca successiva al venir meno dei famosi grands récits, dimostrano soprattutto che l'autrice mira a farlo apparire tale, e a fare i conti, inoltre, con l'epoca precedente e con certe sue ideologie. Non sarà certo questo l'obiettivo principale del romanzo, tuttavia si tratta di una componente da non trascurare ${ }^{12}$.

Che l'epoca non sia più quella delle grandi ideologie sociali traspare del resto anche dal modo di gestire i rapporti interpersonali tenuto dalla protagonista del romanzo : ne abbiamo una conferma anche attraverso un confronto con Porci con le ali, in cui la cellula sociale più importante è il cosiddetto collettivo. È lì che i giovani trovano la loro dose vitale di calore umano, lì sanno di essere al loro posto - anche se succede molte volte che qualcuno "strippi » o che ce l'abbia con quelli un po' più anziani, che la sanno sempre troppo lunga. Diverso il caso di In principio erano le mutande. Una caratteristica importante della protagonista è di non appartenere a nessun "collettivo" del genere. Non solo non è integrata 
nella società borghese (mentre Rocco e Antonia lo sono, perché nonostante le loro velleità rivoluzionarie fanno i bravi liceali), ma non ha nemmeno una sottocultura o controcultura che la accolga. È legata in qualche modo a dei sodalizi artistici, ma questo le serve più che altro per sbevazzare e sfamarsi alle loro feste. Anche in quelle occasioni, peraltro, dimostra un atteggiamento di disincanto ironico nei confronti di chiunque le presenti un'ideologia o una teoria di sorta.

\section{Autocoscienza femminile}

È da qualificare post-femminista Rossana Campo, come sostiene Stefania Lucamante $^{13}$ ? Lo è il personaggio principale di questo suo romanzo ? Confesso che avverto un certo disagio a impiegare questo termine, dato che mi pare ambiguo. C'è chi lo usa per indicare un atteggiamento di rinuncia, di backlash volontario (cioè, di un ritorno al modello femminile tradizionale, in certi casi accompagnato da un fare ironico o sprezzante nei confronti dello stesso femminismo), mentre altri lo usano per caratterizzare coloro che del "femminismo storico" (quello militante e politico degli anni '70) accettano le conquiste, pur non avvertendo più l'esigenza di lottare per la causa della donna. C'entra inoltre la famosa distinzione tra il femminismo della parità e quello della differenza, che complica ulteriormente le cose. Dunque, in che senso del termine il personaggio può definirsi postfemminista ? Come si definisce il suo rapporto con il femminismo e la propria identità femminile?

$\mathrm{E}$ interessante osservare a questo proposito che il movimento femminista degli anni '70 e i suoi slogan non vengono mai ironizzati con battute analoghe a quella degli «espropri rivoluzionari». Mancano d'altronde anche riferimenti "seri» che invece sono ben presenti negli altri testi dell'autrice, soprattutto in Mai sentita così bene, dove si trovano diversi rimandi a teoriche del femminismo come Simone de Beauvoir o Betty Friedan. Inoltre, le «conquiste» politiche del movimento ottenute negli anni '70 vengono date per scontate, e lo stesso vale per certi mutamenti del clima sociale, come l'accettabilità del rapporto di convivenza. La stessa protagonista di In principio erano le mutande non dimostra nessuna voglia né di continuare quelle battaglie ideologiche e politiche, né di assumersi quelle responsabilità che derivano dalle «mete raggiunte» sopra ricordate : non cerca di «realizzarsi» tramite un'attività professionale qualificata, ma si accontenta di arrangiarsi - vale a dire di sbarcare il lunario alla men peggio. Inoltre, non prova nessun bisogno di lottare per la propria indipen- 
denza, perché indipendente lo è già. L' «impegno di rivisitazione dei moduli femministici ${ }^{14}$ " che la Lucamante considera una costante dell'opera campiana, in questo primo romanzo si manifesta, semmai, soltanto tra le righe.

L'essenza della femminilità del personaggio va cercata invece su un altro livello, ovvero quello a cui allude il titolo del romanzo. Silvia Contarini osserva, a proposito di Mai sentita così bene, che "il femminismo di Campo è quello dell'emancipazione sessuale ${ }^{15}$ ». Tale valutazione può essere applicata anche a In principio erano le mutande, con l'unica differenza che, mentre nel libro commentato dalla Contarini questa posizione emerge soprattutto nelle discussioni accese tra amiche, nel primo romanzo viene esclusivamente dimostrata in actu. Tutto sommato, la protagonista del testo coltiva una concezione prettamente edonistica della vita, in cui un ruolo principale è assegnato alla sensualità (che include, oltre al sesso, anche "le gioie del mangiare e del bere», PM 41) e le passioni. È soprattutto amando che afferma la propria femminilità ${ }^{16}$.

Tuttavia va fatta una precisazione importante : ciò che dà sapore e senso all'esistenza della protagonista di In principio erano le mutande non è il piacere solamente carnale, ma le «storie d'amore con tantissima passione e però anche molta tragedia» (PM 28). Gli amori veri e propri meglio ancora se conditi con dei contorni romanzeschi - vengono preferiti agli sfoghi puramente sensuali (benché quest'ultimi non vengano disprezzati). Nell'autodefinizione del personaggio il cuore e anche gli strazi del medesimo hanno sicuramente una parte più importante rispetto alle parti inferiori del corpo.

Una caratteristica essenziale del suo modo di amare è inoltre che anche questo si può definire postideologico. La giovane donna non si dà o non si nega per conformarsi a degli ideali - «l'amore libero » o la ribellione della donna contro lo sciovinismo maschile - ma appunto per il suo piacere carnale o sentimentale. Questo elemento va dunque annoverato tra quelli che abbiamo analizzato nel capitolo precedente.

Torniamo per un attimo a Porci con le ali. Volendo definire questo libro come spesso si fa - il manifesto di una generazione, rimane da chiedersi a quale generazione ci si riferisca. Certo non a quella sessantottina (la quale costituisce per i protagonisti pur sempre un irraggiungibile punto di riferimento), ma neanche a quella di coloro che in seguito sarebbero stati i protagonisti del Settantasette : anagraficamente, i conti non tornano ; i due liceali protagonisti sono semplicemente troppo giovani. Il libro anti- 
cipa tuttavia qualche componente importante di quel movimento : non solo l'importanza data allo "stare insieme», ma anche l'insofferenza nei confronti della sinistra istituzionalizzata - sia quella dei sindacati e del PCI di Berlinguer, ormai avviato sulla strada del compromesso storico (questa parte è recitata nel libro dal padre di Rocco), sia quella dei vari gruppetti extraparlamentari del post-sessantotto. Detta insofferenza si esprime soprattutto nel modo in cui certi personaggi appartenenti a quei gruppi vengono descritti : il ventottenne Carlo che «rappresenta la linea nera, marxista-leninista, paleo-sciovinista ${ }^{18}{ }_{»}$; oppure Marcello, teorico di musica pop e autore del saggio "Zappa e Reich ", ironicamente qualificato come «Dirigente-nazionale-di-organizzazione-culturale vicina all'area di Classe. Praticamente Dio» (PA 38). Dunque, la generazione protagonista di Porci con le ali è quella dei «fratelli più piccoli", più giovani di qualche anno dei sessantottini e dei futuri «settantasettini" : un elemento tra i più importanti nel testo è infatti il rapporto difficile con $\mathrm{i}$ «fratelli maggiori», ideologicamente più preparati e che, all'occorrenza, si pongono meno problemi a soddisfare le proprie voglie di godere ${ }^{19}$. Quest'osservazione ci porta a quello che costituisce forse il lato più interessante del libro. Gli adolescenti sentono infatti un crescente disagio nei confronti sia dei modi di fare dei compagni più anziani sia della permeazione ideologica della loro vita sentimentale. Ciò riguarda più che altro Antonia, la quale avverte un disgusto palese nei confronti del comportamento dei maschi più esperti, per esempio di quello del già menzionato Carlo che la rimorchia, si accoppia sbrigativamente con lei (senza neanche simulare un bricciolo di trasporto), per poi ritornare a giocare a carte con i suoi amici. Ma la protagonista se la prende anche con Rocco quando fa delle asserzioni come : «Io e te stiamo benissimo, ma dobbiamo fare attenzione a non separarci dai compagni. Perché è una cosa borghese» (PA 90) ${ }^{20}$. Ma simili critiche, incentrate sul sospetto della presenza di residui dell'ideologia borghese, vengono rivolte anche allo stesso Rocco, ad esempio quando Marcello per convincerlo ad atti omosessuali aggira agilmente le sue possibili resistenze qualificandole in anticipo come «residuati» di un'educazione restrittiva (PA 46). Inoltre, delle fantasie da romanzo d'appendice di cui si è fatto menzione sopra, almeno quelle che Rocco e Antonia mettono in scena insieme per aggiungere sapore a certi incontri erotici sono interpretabili come tentativi di sottrarsi al dettato dell'ideologia imperante che all'insegna dell'Amore libero condanna il rapporto di coppia perché ritenuto repressivo e dannoso per il collettivo (come emerge anche dalla dichiarazione di Rocco appena citata). La rivendicazione della felicità nel rapporto d'amore da parte di Rocco e Antonia rimane, ahimé, 
episodica, legata a quel breve periodo in cui i due sono uniti davvero da legami sentimentali oltre che erotici. Proprio durante la discussione che pone fine al loro rapporto, Antonia polemizza anche contro la «coppia come istituzione»(PA 122). Infine, quasi tutti i tentativi di ribellione all'ideologia che abbiamo ricordato sono accompagnati da notevoli sensi di colpa.

Il carattere repressivo del dictat de "Il privato è politico" - e anche la volontà dei protagonisti di liberarsene - diventa particolarmente evidente quando si arriva alla questione della procreazione. È proprio nella fase più felice del loro rapporto che Rocco e Antonia sognano di stare insieme a lungo e di avere dei figli - nonostante la lettura de La morte della famiglia di Daniel Cooper sia d'obbligo - e anche di non doverli battezzare Vladimir Ilic. In questo caso l'impulso di ribellione si manifesta in modo particolare, perché persegue un duplice obiettivo, essendo diretto sia contro i teorici della "morte della famiglia» e dei "funerali della coppia» (PA 93), sia contro il comunismo tradizionale e di partito. D'altronde anche qui i ragazzi si sentono in colpa per aver covato tali desideri di felicità personale.

Nel romanzo della Campo invece i figli si fanno sul serio. L'argomento «maternità " affiora più volte nel corso del romanzo, anche ben prima del colpo di scena finale. E non viene taciuto il carattere ambivalente della fecondità : la gravidanza involontaria della protagonista è vista inizialmente come «l'ultima bella botta dell'infame destino» (PM 133), ma porterà anche, dopo qualche rapida peripezia, all'unione felice con l'uomo desiderato, con la prospettiva che il tempo delle "sfighe cosmiche» (PM 42) e della "vita di grandi miserie» (PM 11) sia finito. Rimane però, per il momento, la nausea.

A scorrere un po' la produzione successiva dell'autrice, può sembrare che la questione l'abbia addiritura in qualche modo ossessionata : in Mai sentita così bene la rivelazione di una maternità indesiderata dà avvio al «finale furioso" della vicenda narrata, mentre ne Il matrimonio di Maria e in Mentre la mia bella dorme gravidanze involontarie costituiscono persino la base della trama. In quegli altri libri però si propone un'imma-gine marcatamente disincantata del problema : tanto, i padri sono sempre o dei deficienti o degli scellerati, e in nessuno dei casi si giunge ad una se-rena convivenza dei futuri genitori. In principio erano le mutande invece concede al padre l'occasione di guadagnarsi la qualifica di «ex infame» (PM 145) e di assumersi la responsabilità ; il romanzo finisce con una "scena d'amore bellissima come nei film» (PM 140), dunque con un happy end il cui carattere artificiale e di citazione viene esplicitamente sottolineato. 
Questo capitolo finale del romanzo merita tuttavia uno sguardo un po' più approfondito. Prima di finire in armonia e abbracci, il colloquio della protagonista con il suo amante prende una piega assai diversa, in quanto la giovane donna dimostra inizialmente un atteggiamento molto scontroso : "prendo a gridare [...] : guarda che non mi aspetto nie-e-enteeee da teeeee guarda che questi sono fa-a-ttiiii mieiii... [...] Io e il mio figlio non abbiamo niente da spartiiiireee con un maiale della tua specieeee..." (PM 142 ; nel testo originale, le parole urlate sono tutte in maiuscolo). La gravidanza involontaria le offre la possibilità di giocare con ruoli diversi e di recitare la parte della donna forte e che farà da sé, prima di passare a quella dell'amante dolce e remissiva.

La svolta conclusiva della storia potrebbe essere interpretata come espressione di una posizione post-femminista nel senso 'rinunciatario' del termine, come un elogio della maternità che ponga fine alla vita di godimenti insensati e disorientati - come se il senso di tutte le peripezie narrate precedentemente fosse quello di condurre la protagonista ad accettare il ruolo della brava madre e moglie. Ma questa interpretazione appare poco convincente. Il testo invita infatti a diffidare del carattere positivo di questo lieto fine nella misura in cui, appunto, si sottolinea il suo carattere artificiale e romanzesco : persino gli stessi personaggi affermano nel loro dialogo di sentirsi come dentro un romanzo della categoria di «quelli che vanno a finire bene» (PM 145). Dato che da queste battute i due senza perdere tempo passano ad un amplesso dai toni decisamente erotici, si vede che la maternità non sostituisce la sensualità ma vi convive. La fecondità è dunque - lungi dall'essere ideologicamente caricata - uno dei diversi aspetti della carnalità e dell'identità femminile.

Porci con le ali costituisce un tentativo di rivendicare il diritto alla felicità personale contro i vincoli di certi ideali rivoluzionari che condannano tale individualismo ; tentativo che rimane tuttavia a metà strada. Pur non essendo questa l'intenzione principale degli autori, il libro mette pure in evidenza certe ipocrisie e contraddizioni della cultura giovanile di sinistra dell'epoca e, soprattutto, il carattere in ultima istanza repressivo di certa ideologia liberalizzante. In principio erano le mutande invece è ambientato in un periodo storico che questa ideologia l'ha lasciata alle spalle. Perciò l'autrice ci può presentare un personaggio che si prende appunto quelle libertà che Rocco e Antonia si potevano soltanto sognare. 


\section{Notes}

1. Vedi F. Balletta, 'Weibs-Bilder' : Zwischen Sprach/Witz und Engagement. Ein Gespräch mit Rossana Campo, in "Zibaldone. Zeitschrift für italienische Kultur der Gegenwart» 34 (Herbst 2002), p. 27-35, p. 35.

2. R. Campo, In principio erano le mutande, Milano, 1992, p. 39. Citazioni da questo romanzo d'ora in poi con la sigla PM e l'indicazione della pagina fra parentesi nel testo.

3. Appare curioso che il numero successivo dello stesso periodico presenti una seconda recensione severa - benché più mitigata nei toni - dedicata allo stesso romanzo, nella quale Angelo Guglielmi parla di «linguaggio che riflette [...] l'afasia dei giovani di oggi» (A. Guglielmi, $I l$ romanzo : belle mutande ma senz'anima, in «L'Espresso », XXXVIII-25, 21 giugno 1992, p. 96-97, p. 97.

4. M. Berisso, Livelli linguistici e soluzioni stilistiche. Sondaggi sulla nuova narrativa italiana 1991-1998, in "Lingua e stile», XXXV-3, settembre 2000, p. 471-504.

5. G. Conte, Wie übersetzt man die 'schriftliche Mündlichkeit' von Rossana Campo ? in "Zibaldone. Zeitschrift für italienische Kultur der Gegenwart», 34, Herbst 2002, p. 37-48.

6. C. Litherland, Rossana Campo : Un-learning the Rules of Writing, in "The Italianist», XXIV1, 2004, p. 126-134, p. 128.

7. Ibid.

8. Ibid., p. 129.

9. Nella già ricordata scena iniziale del romanzo si legge la domanda rivolta al pubblico : «Mi giro e sapete allora chi è ?», la quale ricorda il famoso « '...un re!' diranno i miei piccoli lettori» del Pinocchio.

10. Qui si può osservare un parallelo interessante con Porci con le ali : anche in quel testo, i due adolescenti nei loro incontri amorosi (o per far partire le fantasie masturbatorie) ogni tanto s'immaginano vere e proprie scene da film o da romanzo d'appendice, con personaggi come il famoso avventuriere e spadaccino conte Vronski. Vedremo in seguito che questo fatto è più significativo di quanto possa sembrare a prima vista.

11. Tesi che sarebbe peraltro difficile da sostenere nel caso degli ormai ben sei romanzi ambientati all'estero.

12. In Mentre la mia bella dorme (1999) questa intenzione dell'autrice è ben più vistosa e decisa : in quella specie di detective story, una giornalista si propone di chiarire perché una sua amica poco più che ventenne si sia suicidata senza motivo apparente, e man mano viene a conoscenza di un antefatto complesso. In una comunità rurale hippie nella Francia meridionale degli anni '70 una donna, con una figlia quattordicenne, convive con il proprio amante. Dopo che l'amante ha violentato l'adolescente, la donna fa passare la bambina che nasce da questo stupro come propria figlia. Venti anni dopo, la ragazza scopre l'intera storia e suo padre, divenuto nel frattempo un manager affermato, temendo che sua figlia possa palesare tutto, decide di farla tacere. L'apparente suicidio si rivela dunque essere un assassinio vero e proprio. In quel libro, l'autrice sembra mirare a denunciare le menzogne e la repressione sessuale che si potevano nascondere nell'ideologia love and peace. Nel suo primo romanzo invece, il suo atteggiamento nei confronti del passato è piuttosto beffardo che non accusatorio.

13. Cf. S. Lucamante, 'Una laudevole fine': femminismo e identificazione delle donne nella narrativa di Rossana Campo, in "Italianistica", XXXI-2/3, maggio/dicembre 2002, p. 295-306.

14. Ibid., p. 305.

15. S. Contarini, Riflessioni sulla narrativa femminile degli anni ' 90 , in "Narrativa", 10 , sept. 1996, p. 139-163, p. 157.

16. Va aggiunto che in questo suo modo di concepire e gestire la vita sentimentale e sessuale sta la differenza più notevole tra l'epoca presente e quella dell'adolescenza : anche in quell'epoca remota, il sesso era l'argomento principale delle conversazioni fra le amiche, ma sia quei discorsi, sia i primi tentativi di tresca da parte della ragazza restavano pur sempre l'imitazione di certi modelli di comportamento proposti da altri (per esempio dalle cosiddette "cugine porche" (PM 33) più grandi di qualche anno). La donna adulta invece, attraverso il proprio modo di amare (e di parlare delle proprie esperienze), afferma la sua autonomia e indipendenza. Detti capitoli di retro- 


\section{Peter Gahl}

spettiva assolvono dunque anche la funzione di mettere in particolare evidenza questa evoluzione del personaggio.

17. Nel corso della storia la narratrice consuma talvolta delle esperienze omosessuali. Quando ne parla confidenzialmente con un suo amico - a sua volta bisessuale -, fa una distinzione interessante : dichiara di innamorarsi sia di uomini sia di donne, «ma le grandi passione tragiche solo con i maschi» (PM 80). L'amore lesbico rappresenta dunque la tentazione del godimento puro, senza le sofferenze del cuore, e a un certo punto la giovane donna, momentaneamente delusa dall' "infame» di turno, sta per cedere a questa tentazione : «Forse che dovrò anch'io diventare una donna che si mette lì e ama solo le altre donne simili dello stesso sesso ?» (PM 125). Ma è appunto in virtù di questa sua "concezione tragica della vita" (PM 84) che abbandonerà subito questa strada.

18. Rocco e Antonia [L. Ravera, M. Lombardo Radice], Porci con le ali. Diario sessuo-politico di due adolescenti, Roma, Savelli, 1976 [si cita dalla IX ediz., dic. 1976], p. 161. Citazioni da questo volume d'ora in poi con la sigla PA e l'indicazione della pagina fra parentesi nel testo.

19. In alcune occasioni, il moto di insofferenza dei più giovani si manifesta anche tramite commenti ironici riguardanti certi elementi stilistici propri del gergo politicizzante e sinistroide dei vari «leaderoni» e "leaderini». Se ne trovano per esempio nella descrizione della conferenza sulla musica pop di Marcello (che risulta scarsamente comprensibile al giovane pubblico), il quale usa ripetutamente la locuzione «in ultima analisi», ovvero quella «formula con cui chi ha più di 25 anni sostituisce il 'secondo me' di quando aveva meno di venticinque anni » (PA 41), come chiosa maliziosamente Antonia.

20. Va aggiunto che gli avvenimenti successivi dimostrano che c'è anche una buona dose di ipocrisia in questo atteggiamento da "Monsignor rivoluzione» (PA 90) di Rocco, dato che a stroncare il rapporto di coppia non sarà certo la sua rigorosa condotta politica bensì le sue fissazioni sul piano sessuale, che rappresentano piuttosto un eccesso di intimità. 